
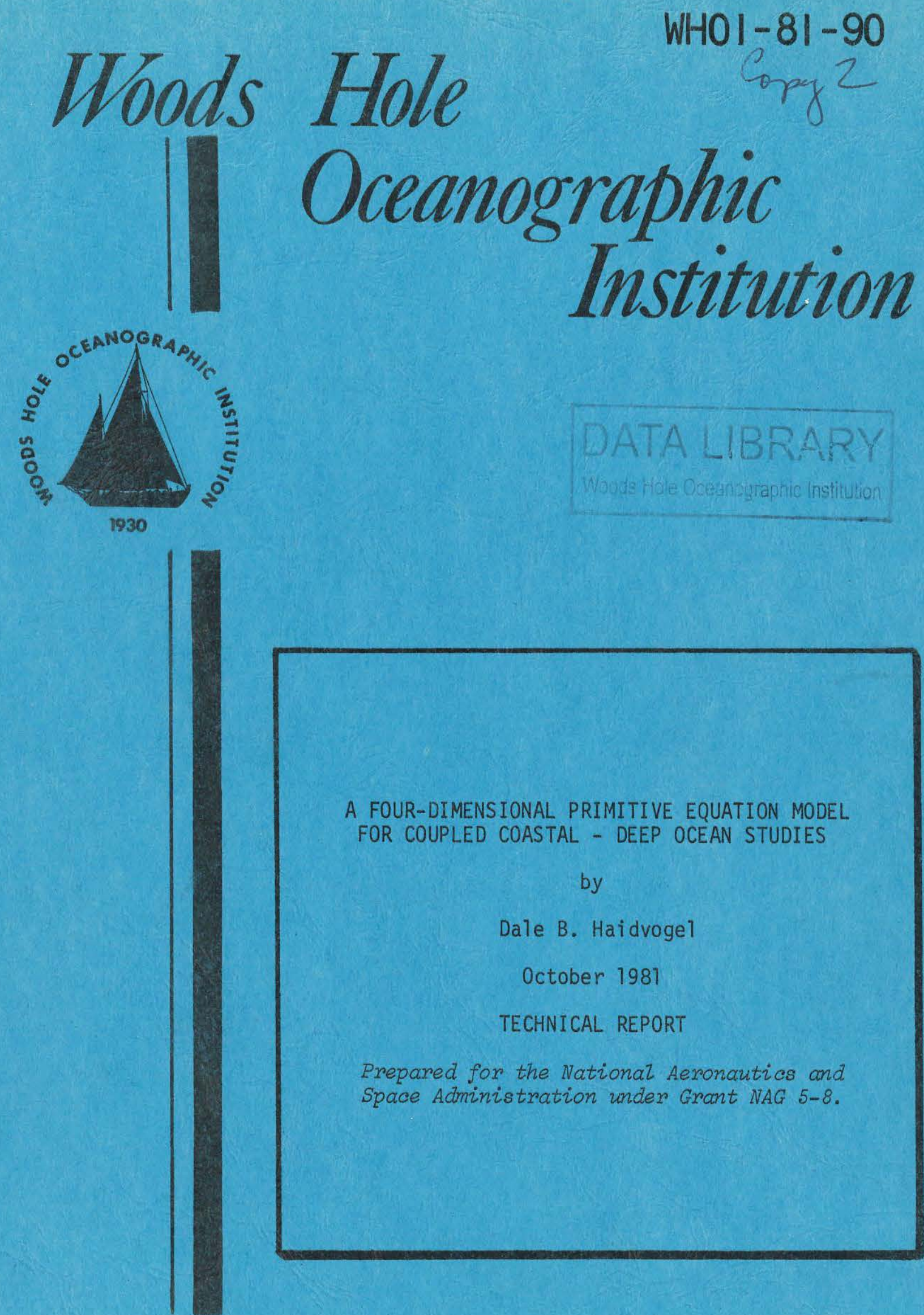

A FOUR-DIMENSIONAL PRIMITIVE EQUATION MODEL FOR COUPLED COASTAL - DEEP OCEAN STUDIES

by

Dale B. Haidvogel

October 1981

TECHNICAL REPORT

Prepared for the National Aeronautics and Space Administration under Grant NAG 5-8.

WOODS HOLE, MASSACHUSETTS 02543

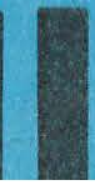


WHOI $-81-90$

\section{A FOUR-DIMENSIONAL PRIMITIVE EQUATION MODEL FOR COUPLED COASTAL - DEEP OCEAN STUDIES}

by

Dale B. Haidvogel

WOODS HOLE OCEANOGRAPHIC INSTITUTION

Woods Hole, Massachusetts 02543

October 1981

TECHNICAL REPORT

Prepared for the National Aeronautics and Space Administration under Grant NAG 5-8.

Reproduction in whole or in part is permitted for any purpose of the United States Government. This report should be cited as: Woods Hole Oceanog. Inst. Tech. Rept. WHOI-81-90.

Approved for Distribution:

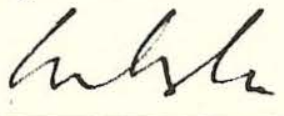

Valentine Worthington, Chairman Department of Physical Oceanography 
TABLE OF CONTENTS

Table Captions

Page

Abstract

1. Introduction

2. Model Equations

3. Sigma (stretched vertical) Coordinate System

4. Numerical Discretization and Solution

5. Model Performance and Evaluation

6. Acknowledgements

21

Table I

22

Table II

23

Appendix

24

References

28 


\section{Table Captions}

Table I: Normalized RMS Model Errors in Streamfunction $(\psi)$ and Velocity $(u, v)$ After One Period for Nonlinear Topographic Wave Solutions.

Table II: Required cpu Time Per Model Time-Step on a VAX11-780. 
ABSTRACT

A prototype four-dimensional $(x, y, z, t)$ continental shelf/deep ocean model is described. In its present form, the model incorporates the effects of finite-amplitude topography, advective nonlinearities, and variable stratification and rotation. The model can be forced either directly by imposed atmospheric windstress and surface pressure distributions, and energetic mean currents imposed by the exterior oceanic circulation; or indirectly by initial distributions of shoreward propagating mesoscale waves and eddies.

To avoid concerns over the appropriate specification of "open" boundary conditions on the cross-shelf and seaward model boundaries, a periodic channel geometry (oriented along-coast) is used. The model employs a traditional finite-difference expansion in the cross-shelf direction, and a Fourier (periodic) representation in the long-shelf coordinate. A modified sigma coordinate system, and a Chebyshev-tau approximation scheme, are used to incorporate the vertical dependence.

The model has been validated against a variety of propagating topographic wave problems. Representative run times and error estimates are given. 


\section{Introduction}

The dynamical coupling between the large-scale ocean circulation and motions on the continental shelf and slope is at present poorly understood; nonetheless, the existence and implications of that coupling are of concern to both coastal and deep-water oceanographers. For example, exterior current systems undoubtedly place strong constraints on the form of the adjacent shelf circulation, thereby dictating circulation patterns in the important near-shore zone. Increasing evidence suggests, for instance, that the observed southwesterly directed mean flow along the northeastern continental shelf of the United States is driven by a longshore pressure gradient supported by the iarge-scale ocean circulation (Csanady, 1978). Conversely, flow along the continental margin can be thought of as a boundary layer component of the exterior ocean circulation (Beardsley and Winant, 1979). A refined description of the closure of open ocean flow by the shelf/slope system would therefore contribute strongly to modeling studies of the wind- and eddy-driven oceanic general circulation [see also, Haidvogel (1979)].

These and other considerations suggest that a detailed knowledge of the operative continental shelf/deep ocean coupling mechanisms is of considerable practical importance. This is not only true, however, from strictly an ocean modeling point of view. As well as being areas of intense mean flow and potentially significant mesoscale eddy/mean field interaction, the western boundaries of the world's oceans are also sites of strong ocean-atmosphere transfers - for example, of heat. The continental slope regions - representing as they do a large reservoir of heat, and 
acting as preferred locations for oceanic frontal formation - may contribute strongly to the maintenance and form of that heat flux. A proper definition of the role of the continental margins in closing the general ocean circulation is ultimately relevant, therefore, to a broad range of questions, including local oceanographic and global air-sea interaction (i.e., climate) problems.

Recent observational evidence in the near-shore and deep ocean regions suggest three plausible mechanisms for shelf/open ocean coupling. The first is the aforementioned mean alongshore pressure gradient (believed to be) imposed at the outer edge of the shelf by the exterior large-scale circulation. Such a pressure gradient, and its extension across the narrow shelf, is thought to drive the mean southwestward flow in the Middle Atlantic Bight and thereby to support the observed shelfslope front (Beardsley and Winant, 1979). Second are nearly linear, low frequency motions--thought to be topographic Rossby waves--which, generated in the deeper ocean, are directed shoreward and propagate onto the continental slope. The most complete description of the properties of these low frequency motions has been synthesized from data taken at Site D, a long-term observing location situated on the continental rise north of the mean axis of the Gulf Stream. Results at Site D indicate most of the low frequency energy to be associated with periods of a week to a few months and with a shoreward energy flux. Thompson (1977) offers a review. Finally, mixing and/or transport of properties across the shelfslope boundary is associated with intense eddy motions impinging on the shelf/slope region from the open ocean. These nonlinear features, though 
intermittent, are strongly energetic and have been observed to account for sizable local heat and momentum exchange [e.g., see Smith (in press)]. Such intense mesoscale features can often be seen, and their penetration of shelf waters monitored, by satellite infrared imagery (Ring Workshop, 1977).

Theoretical and numerical studies of linear topographic Rossby waves incident from the open ocean onto the continental slope have been made (Kroll and Niiler, 1976; Ou, 1979). Results suggest that linear wave mechanisms are unlikely to be quantitatively important in the coupling of shelf and open ocean circulations. At the present time, therefore, the "best-guess" scenario is that: (1) longshore pressure gradients imposed by the exterior circulation are instrumental in supporting mean shelf currents; and (2) energetic eddy motions provide significant transient exchange of heat and momentum across the continental slope.

A numerical model, using primitive equation dynamics, has been developed to begin to investigate the details of this continental shelf/deep ocean coupling. The dynamic equations and physical assumptions of this model are quickly reviewed in Section 2. A sigma coordinate system for the vertical dependence is then introduced (Section 3), and the resulting four-dimensional problem (in $x, y, \sigma$ and $t$ ) is discretized for efficient and accurate solution (Section 4). Test problems are examined in Section 5 to determine model accuracy and cpu requirements.

\section{Model Equations}

The model is governed by the hydrostatic primitive equations, which 
can be written:

$$
\begin{aligned}
& \frac{\partial u}{\partial t}+\underset{\sim}{u} \cdot \nabla u-f v=-\frac{1}{\rho_{0}} \frac{\partial}{\partial x}\left(p+p_{a}\right)+\frac{\partial}{\partial z}\left(v \frac{\partial u}{\partial z}\right) \\
& \frac{\partial v}{\partial t}+\underset{\sim}{v} \cdot \nabla v+f u=-\frac{1}{\rho_{0}} \frac{\partial}{\partial y}\left(p+p_{a}\right)+\frac{\partial}{\partial z}\left(v \frac{\partial v}{\partial z}\right) \\
& \frac{\partial \rho}{\partial t}+\underset{\sim}{u} \cdot \nabla \rho=\frac{\partial}{\partial z}\left(\kappa \frac{\partial \rho}{\partial z}\right) \\
& \frac{\partial p}{\partial z}=-\rho g \\
& \frac{\partial u}{\partial x}+\frac{\partial v}{\partial y}+\frac{\partial w}{\partial z}=0
\end{aligned}
$$

where, in standard notation:

$$
\begin{aligned}
(u, v, w) & =(x, y, z) \text { components of vector velocity } \sim \sim \\
\rho & =\text { density } \\
p_{a} & =\text { imposed (atmospheric) pressure } \\
f & =f_{0}[1+\beta(y)]=\text { Coriolis parameter } \\
g & =\text { acceleration of gravity (acting vertically downwards) }
\end{aligned}
$$

and

$$
\begin{aligned}
(\nu, \kappa)= & \text { vertical diffusive coefficients for horizontal } \\
& \text { momentum and density. }
\end{aligned}
$$

Equations (1) and (2) express the momentum balance in the $x$ and $y$ directions, respectively. The time evolution of the density field, $\rho(x, y, z, t)$, is governed by the advective-diffusive equation (3). Lastly, equations (4) and (5) express the hydrostaticity and incompressibility of the flow field. 
Note that the Boussinesq and rigid lid approximations have been invoked. The latter, in particular, is numerically advantageous in that it effectively filters out surface gravity waves which otherwise place strong restrictions on time-stepping (see Section 4). We do not expect, however, that this assumption will seriously affect the physical representation of the coastal/deep ocean coupling of interest.

For convenience, and to avoid dealing with the considerable problem associated with the specification of appropriate "open" boundary conditions, we choose to work within a periodic channel oriented in the alongcoast direction. The region is bounded above by a rigid lid, and below by a variable bottom of depth $h(x, y)$. The assumption of periodicity in the along-coast direction, and the placement of a fictitious "wall" at some distance from the coast are, of course, only crude approximations to physical reality. Although they allow rather confident and straightforward treatment of equation (1-5), the influence of these assumptions on the modeled flow must be borne in mind. It is expected, however, that the existence of these boundaries will be unimportant if they are placed at a sufficient distance from the sites of coastal/deep ocean interaction.

Consistent with the assumption of the periodic channel geometry, suitable boundary conditions for equations (1-5) are chosen as follows:

$$
\begin{aligned}
& \text { sidewalls }\left(y=0, L_{y}\right) \quad v=0 \\
& \operatorname{top}(z=0) \quad v \frac{\partial u}{\partial z}=\tau{ }_{x}^{0}(x, y) \\
& v \frac{\partial V}{\partial z}=\tau{ }_{y}^{0}(x, y) \\
& \eta \frac{\partial p}{\partial z}=\tau{ }_{p}^{0}(x, y)
\end{aligned}
$$




$$
\text { and bottom }(z=-h) \quad \begin{array}{rlrl}
v \frac{\partial u}{\partial z} & =\tau \frac{h}{x}(x, y) & & \text { or } u=0 \\
v \frac{\partial v}{\partial z} & =\tau \frac{h}{y}(x, y) & \text { or } v=0 \\
\eta \frac{\partial \rho}{\partial z} & =0 . &
\end{array}
$$

Thus, the sidewalls are required to be impermeable (equation 6 ), and surface distributions of wind stress $\left(\tau_{x}^{0}, \tau_{y}^{0}\right)$ and density flux $\left(\tau_{p}^{0}\right)$ are prescribed on the top (equation 7). On the variable bottom, $z=-h(x, y)$, the horizontal velocity components are constrajned either to vanish, or to accommodate a prescribed bottom stress; the density flux is taken to vanish. It is relevant to note at this point that the vertical (Chebyshev-tau) discretization scheme to be described in next section can trivially accommodate quite arbitrary boundary conditions on $z=0,-h$. Lastly, all variables are required to be periodic over the interval $0 \leq x \leq L_{x}$

\section{Sigma (stretched vertical) Coordinate System}

From the point of view of the computational model, it is highly convenient to introduce a stretched vertical coordinate system, which essentially "flattens out" the variable bottom at $z=-h(x, y)$. Such "sigma" coordinate systems have long been used, with slight appropriate modification, in both meteorology and oceanography [e.g., Phillips (1957) and Freeman et al. (1972)]. To proceed, we make the coordinate transformation:

$$
\begin{aligned}
& \hat{x}=x \\
& \hat{y}=y \\
& \sigma=1+2(z / h)
\end{aligned}
$$


and

$\hat{t}=t$.

In the stretched system, the vertical coordinate $\sigma$ spans the range $-1 \leq \sigma$ $\leq 1$; we are therefore left with a domain of regular shape. As a trade-off for this geometric simplification, however, the dynamic equations become somewhat more complicated. The resulting dynamic equations are, dropping the carats:

$$
\begin{aligned}
& \frac{\partial u}{\partial t}-f v=-\underset{\sim}{v} \cdot \nabla u-\frac{g}{2 \rho_{0}}\left[\frac{\partial}{\partial x}\left(h \int_{\sigma}^{1} \rho d \sigma\right)-(1-\sigma) \rho \frac{\partial h}{\partial x}\right] \\
& -\frac{1}{\rho_{0}} \frac{\partial}{\partial x} p^{0}+\frac{4}{h^{2}} \frac{\partial}{\partial \sigma}\left(v \frac{\partial u}{\partial \sigma}\right) \\
& \frac{\partial v}{\partial t}+f u=-\underset{\sim}{v} \cdot \nabla v-\frac{g}{2 \rho_{0}}\left[\frac{\partial}{\partial y}\left(h \int_{\sigma}^{1} \rho d \sigma\right)-(1-\sigma) \rho \frac{\partial h}{\partial y}\right] \\
& -\frac{1}{\rho_{0}} \frac{\partial}{\partial y} p^{0}+\frac{4}{h^{2}} \frac{\partial}{\partial \sigma}\left(\nu \frac{\partial v}{\partial \sigma}\right) \\
& \frac{\partial \rho}{\partial t} \quad=-\underset{\sim}{v} \cdot \nabla \rho \quad+\frac{4}{h^{2}} \frac{\partial}{\partial \sigma}\left(\kappa \frac{\partial \rho}{\partial \sigma}\right) \\
& \frac{\partial}{\partial x}[h u]+\frac{\partial}{\partial y}[h v]+h \frac{\partial W}{\partial \sigma}=0
\end{aligned}
$$

where the vertical velocity in sigma coordinates:

$$
w(x, y, \sigma, t)=\frac{1}{h} \quad\left[(1-\sigma) u \frac{\partial h}{\partial x}+(1-\sigma) v \frac{\partial h}{\partial y}+2 w(x, y, z, t)\right] .
$$

$\mathrm{p}^{0}$ is the net pressure at $z=0$ - the sum of the imposed atmospheric pressure and the "reaction pressure" due to the presence of the rigid lid. Note also that the dynamic pressure has been eliminated from the equations using the hydrostatic relation. 
In the stretched coordinate, boundary conditions (6-8) become:

$$
\begin{aligned}
& \text { sidewalls }\left(y=0, L_{y}\right) \quad v=0 \\
& \text { top }(\sigma=1) \quad\left(\frac{2 v}{h}\right) \frac{\partial u}{\partial \sigma}=\tau{ }_{x}^{0}(x, y) \\
& \left(\frac{2 v}{h}\right) \frac{\partial v}{\partial \sigma}=\tau \frac{0}{y}(x, y) \\
& \left(\frac{2 \eta}{h}\right) \frac{\partial \rho}{\partial \sigma}=\tau{ }_{\rho}^{0}(x, y) \\
& \text { and bottom }(\sigma=-1) \\
& \left(\frac{2 v}{h}\right) \frac{\partial u}{\partial \sigma}=\tau{ }_{x}^{h}(x, y) \quad \text { or } u=0 \\
& \left(\frac{2 v}{h}\right) \frac{\partial v}{\partial \sigma}=\tau \frac{h}{y}(x, y) \quad \text { or } v=0 \\
& \left(\frac{2 \eta}{h}\right) \frac{\partial \rho}{\partial \sigma}=0
\end{aligned}
$$

Details of this coordinate transformation have been given by several authors - see, for instance, Owen (1980).

4. Numerical Discretization and Solution

(4a) Chebyshev Polynomial Basis Set

In the vertical $(\sigma)$ direction, it is dynamically appropriate and computationally convenient to represent the motion in terms of a sum of its depth-averaged (external) and depth-varying (internal) components. To do so, we represent the vertical dependence of the dependent variables as an expansion in the polynomial set $P_{k}(\sigma)$ :

$(u, v, \rho, w)=\sum_{k=0}^{N}\left[u_{k}(x, y, t), v_{k}(x, y, t), \rho_{k}(x, y, t), w_{k}(x, y, t)\right] P_{k}(\sigma)$.

We further require that $\delta_{-1}^{1} P_{k}(\sigma)$ do $=\delta_{0 k}$ - that is, that only the zeroeth 
order polynomial have a non-zero vertical integral.

The $P_{k}(\sigma)$ expansion functions can be chosen somewhat arbitrarily. We choose to use a modified form of the Chebyshev polynomial of the first kind. In particular, we set:

$$
P_{k}(\sigma)= \begin{cases}\frac{1}{2} T_{0}(\sigma) & k=0 \\ T_{k}(\sigma) & k \geq 1, k \text { odd } \\ T_{k}(\sigma)+\frac{1}{k^{2}-1} & k \geq 2, k \text { even. }\end{cases}
$$

Note that the $P_{k}(\sigma)$ conform to the required integral property; further, since $P_{0}(\sigma)$ is identically constant with depth, it represents the depthaveraged component of the field.

Further properties of the Chebyshev polynomial basis functions are summarized in the Appendix.

(4b) Discretization in $x$ and $y$

Adopting a complex Fourier expansion in $x$, and a finite-difference representation in $y,(17)$ becomes:

$$
\begin{aligned}
& (u, v, \rho, w)_{1 m n}=\underset{j=-L / 2}{L / 2-1} \sum_{k=0}^{N}\left[u_{j m k}(t), v_{j m k}(t), \rho_{j m k}(t), w_{j m k}(t)\right] \\
& \begin{array}{lll}
e^{-2 \pi j x_{1} / L} \times & & 0 \leq 1 \leq L \\
& P_{k}(\sigma) & 0 \leq m \leq M \\
& 0 \leq n \leq N
\end{array}
\end{aligned}
$$

where, for instance, $u_{1 m n}=u\left(x_{1}, y_{m}, \sigma_{n}, t\right)$. The locations of the equivalent "grid-points" associated with this expansion are given by:

$$
\begin{aligned}
& x_{1}=1 L_{x} / L \\
& y_{m}=m L_{y} / M
\end{aligned}
$$


and

$$
\sigma_{n}=\cos [\pi(N-n) / N] .
$$

It should be noted that the assumed reality of the fields $(u, v, \rho, w)$ implies the associated conjugate symmetry condition:

$$
\left[u_{j}, v_{j}, \rho_{j}, w_{j}\right]_{m k}=\left[u_{-j}^{*}, v_{-j}^{*}, \rho_{-j}^{*}, w_{-j}^{*}\right]_{m k}
$$

(4c) Evaluation of Spatial Derivatives and Vertical Integrals In representation (19), the semi-discrete form of the dynamic equations (10-13) can be written:

$$
\begin{aligned}
\frac{\partial u}{\partial t}-f v=-\left(u \delta_{x} u+v \delta_{y} u+\right. & \left.w_{\sigma} u\right)-\frac{g}{2 \rho_{0}}\left[\delta_{x}\left[h I_{\sigma}^{1}(\rho)\right]-(1-\sigma) \rho \delta_{x} h\right]-\frac{1}{\rho_{0}} \delta_{x} p^{0} \\
& +\frac{4}{h^{2}} \delta_{\sigma}\left(v \delta_{\sigma} u\right) \\
& =A
\end{aligned}
$$

$$
\begin{aligned}
\frac{\partial v}{\partial t}+f u=-\left(u \delta_{x} v+v \delta_{y} v+\right. & \left.w \delta_{\sigma} v\right)-\frac{g}{2 \rho_{0}}\left[\delta_{y}\left[h I_{\sigma}^{1}(\rho)\right]-(1-\sigma) \rho \delta_{y} h\right]-\frac{1}{\rho} \delta_{y} p^{0} \\
& +\frac{4}{h^{2}} \delta_{\sigma}\left(v \delta_{\sigma} v\right) \\
& =B
\end{aligned}
$$

$\frac{\partial \rho}{\partial t}=-\left(u \delta_{x} \rho+v \delta_{y} \rho+w \delta_{\sigma} \rho\right)+\frac{4}{h^{2}} \delta_{\sigma}\left(k \delta_{\sigma} \rho\right)$

and

$$
\begin{gathered}
=C \\
\delta_{x}(h u)+\delta_{y}(h v)+\delta_{\sigma}(h w)=0,
\end{gathered}
$$

where it is understood that the equations are to be evaluated at the gridpoints $\left(x_{1}, y_{m}, \sigma_{n}\right)$; or alternately, in horizontal gridpoint/vertical mode space. 
Here, $\delta_{x}, \delta_{y}$ and $\delta_{\sigma}$, and $I_{\sigma}^{1}(\rho)$ denote the discrete forms of the differential and integral operators occurring in the continuum equations (1013). They are evaluated as follows:

(i) $\delta_{x}$ : Derivatives in the $x$-direction are computed by direct Fourier synthesis using a one-dimensional discrete Fast Fourier Transform (FFT). Thus, a forward transform, followed by a multiplication of the resulting Fourier spectrum by $\left(-2 \pi i j / L_{y}\right)$ and lastly, an inverse transform yields the desired $x$ derivative.

(ii) $\delta_{y}: \quad$ Derivatives in the y-direction are computed using the traditional centered, second-order finite-difference approximation, except at the edges of the domain (the channel walls) where an uncentered (one-sided) second-order formula is used. and $(i i i) \delta_{y}$ and $I_{\sigma}^{1}(\rho)$ :

Both derivatives and integrals in $\sigma$ can be implemented by constructing the appropriate matrix transformation - i.e., for instance, the matrix which transforms a given vertical column of point values into the values of the derivatives at those points. These transformation matrices are easily constructed by reference to the known properties of the Chebyshev basis functions $P_{k}(\sigma)$. The structure of these matrices is discussed in the Appendix.

(4d) Time-Stepping: Depth-Integrated Transport

Taking $k=0$, equations (20-23) describe the time evolution of the depth-integrated flow field. In particular - since $W_{1 m 0}(t) \equiv 0$; i.e., the depth-averaged vertical velocity vanishes - the $k=0$ transport field 
$\left[\mathrm{hU}_{1 \mathrm{~m} 0}, \mathrm{hV}_{1 \mathrm{~m} 0}\right]$ is horizontally non-divergent by (23). Taking advantage of this property, we introduce the horizontal mass transport streamfunction $\psi_{1 \mathrm{~m}}(t)$, where

$$
[u(t), v(t)]_{1 m 0}=\left[-\delta_{y} \psi, \delta_{x} \psi\right]_{l m} .
$$

Taking the curl of the momentum equations (20) and (21) in the usual way, we obtain a vorticity equation for the depth-integrated flow field:

$$
\begin{gathered}
\frac{\partial}{\partial t} \zeta_{1 m}=\frac{\partial}{\partial t}\left[\delta_{x} \delta_{x} \psi+\delta_{y} \delta_{y} \psi-\frac{1}{h}\left(\delta_{x} h\right) \psi_{x}-\frac{1}{h}\left(\delta_{y} h\right) \psi_{y}\right]_{1 m}= \\
h_{1 m}\left[\delta_{x}\left(\frac{f}{h}\right) \psi_{y}-\delta_{y}\left(\frac{f}{h}\right) \psi_{x}+\delta_{x} B_{0}-\delta_{y} A_{0}\right]_{1 m} \\
=D_{1 m} .
\end{gathered}
$$

Note that $\left(A_{0}\right)_{1 m}$ and $\left(B_{0}\right)_{1 m}$ are the depth-integrated components of the cumulative right-hand side terms in equations (20) and (21). This comporient is obtained from the associated physical space (vertical) distribution of values by a simple matrix transformation - see the Appendix. Not only does the introduction of $\psi$ automatically guarantee horizontal nondivergence, but it eliminates the pressure gradient term associated with the (unknown) reaction pressure supported by the rigid lid. A solution to equation (24) is fully prescribed by specifying the (constant) boundary values of $\psi$ on the channel walls - that is, by setting:

$$
\psi_{10}(t)=\psi_{0}(t) \text { and } \psi_{1 M}(t)=\Psi_{M}(t) \text {. }
$$

The vorticity equation (24) is advanced in time using a second-order Adams-Bashforth approximation. The resulting fully discrete equation is: 


$$
\zeta{ }_{1 m}^{q+1}=\zeta{ }_{1 m}^{q}+\left(\frac{3 \Delta t}{2}\right) D_{1 m}^{q}-\left(\frac{\Delta t}{2}\right) D_{1 m}^{q-1}
$$

where $q$ is the time step index. By applying a forward transform in $x$, the Helmholtz-1ike equation (25) - and associated boundary conditions (25) can be reduced to a sequence of $(M-2) \times(M-2)$ complex triagonal matrix equations, one for each Fourier component $(j)$ in the $x$ expansion. These triagonal equations can be efficiently solved to yield the updated streamfunction field $\psi_{1 \mathrm{~m}}^{\mathrm{q}+1}$.

(4e) Time-Stepping: Internal Velocity Modes ( $1 \leq \mathrm{k} \leq \mathrm{N}-2)$

The (N-2) lowest order internal modes of the velocity distribution that is, $(u, v)_{1 m k}$ for $1 \leq k \leq N-2-$ can be obtained by direct time-stepping of equations (20 and (21). Using an Adams-Bashforth time-step on the right-hand side terms $A$ and $B$, and a Crank-Nicolson approximation on the Coriolis terms, we get:

$$
\begin{gathered}
{\left[u-\left(\frac{f \Delta t}{2}\right) v\right]_{1 m k}^{q+1}=\left[u+\left(\frac{f \Delta t}{2}\right)\right]_{1 m k}^{q}+\left(\frac{3 \Delta t}{2}\right) A^{q}-\left(\frac{\Delta t}{2}\right) A^{q-1}} \\
=G_{1 m k} \\
{\left[v+\left(\frac{f \Delta t}{2}\right) u\right]_{7 m k}^{q+1}=\left[v-\left(\frac{f \Delta t}{2}\right)\right]_{1 m k}^{q}+\left(\frac{3 \Delta t}{2}\right) B^{q}-\left(\frac{\Delta t}{2}\right) B^{q-1}} \\
=H_{1 m k}
\end{gathered}
$$

where the indices $0 \leq 1 \leq L, 0 \leq m \leq M$ and $1 \leq k \leq N-2$. The straightforward algebraic solution to these equations is:

and

$$
\begin{aligned}
& u_{1 m k}^{q+1}=(G+\alpha H)_{1 m k} /\left(1+\alpha^{2}\right) \\
& v_{7 m k}^{q+1}=(H-\alpha G)_{1 m k} /\left(1+\alpha^{2}\right)
\end{aligned}
$$


where $\alpha=f \Delta t / 2$.

(4f) Time-Stepping: Density Equation $(0 \leq k \leq N-2)$

The $0 \leq k \leq N-2$ vertical modes of the density field are advanced in time using equation (22). Without further elaboration, the resulting fully discrete form of (22) is:

$$
\rho_{1 \mathrm{mk}}^{q+1}=\rho_{\text {Imk }}^{q}+\left(\frac{3 \Delta t}{2}\right) c_{1 \mathrm{mk}}^{q}-\left(\frac{\Delta t}{2}\right) c_{1 \mathrm{mk}}^{q-1}
$$

where $0 \leq 1 \leq L, 0 \leq m \leq M$ and $0 \leq k \leq N-2$.

(4g) Application of Boundary Conditions Using the Tau Method Note, in Sections (4d, e, f) that only the lowest (N-1) modes of $(u, v, p)^{q^{+1}}$ have been obtained by time integration of the dynamic equations (20-22). The remaining two highest vertical modes - that is to say, $(u, v, p))_{7 m k}^{q+1}$ with $0 \leq 1 \leq L, 0 \leq m \leq M$ and $N-1 \leq k \leq N$ - are chosen such that the resulting $(u, v, p)^{q+1}$ fields identically satisfy the prescribed boundary conditions on $\sigma= \pm 1$ (equations 15-16). This is the so-called tau method, and can be shown to deliver solutions of comparable accuracy to those obtained using Galerkin or collocation approximation techniques. Definitions, further discussion, and sample comparisons of these spectral approximation techniques has been given by Gottlieb and Orszag (1977).

Having obtained $(u, v, \rho){ }_{1 m k}^{q+1}$ for the lower vertical modes $0 \leq k \leq N-2$, the solution of a simple algebraic problem at each horizontal location $\left(x_{1 m}, y_{1 m}\right)$ provides the required values of $(u, v, \rho)_{1 m k}^{q+1}$ for $k=N-1, N$. The precise structure of these algebraic equations is discussed in the Appendix. (4h) Determination of the Vertical Velocity Field Having obtained a complete specification of the $u, v$, and $\rho$ fields at 
time step $(q+1)$ by the methods outlined above, the vertical velocity field at the interior vertical gridpoints $\sigma_{n}(1 \leq n \leq N-1)$ can be computed:

$$
w_{1 m n}^{q+1}=\frac{1}{h_{1 m}} I_{\sigma_{n}}^{1}\left[\delta_{x}\left(h u_{1 m n}\right)+\delta_{y}\left(h v_{1 m n}\right)\right]
$$

where $0 \leq 1 \leq L, 0 \leq m \leq M$ and $1 \leq n \leq N-1$. In the sigma coordinate system, $w_{1 \mathrm{mO}}=w_{1 \mathrm{mN}} \equiv 0$.

\section{Model Performance and Evaluation}

The numerical model described in the preceding sections has been validated on a variety of simple test problems for which analytic solutions are available. Results for one such representative class of tests are given next.

With uniform rotation and density $\left(f=f_{0}\right.$ and $\left.\rho \equiv 1\right)$, and with the exponential bottom profile $h(x, y)=H_{0} e^{-2 \alpha y}$, nonlinear topographic wave solutions of equations (20-24) can be found. The solutions are depthinvariant; hence, they can be written explicitly in terms of the transport streamfunction. The analytic solution is:

$$
\psi_{a}(x, y, t)=e^{-\alpha y} \sin \left(\pi j y / L_{y}\right) e^{i(k x-\omega t)},
$$

with the accompanying dispersion relation

$$
\omega=-2 f_{0} \alpha k /\left(k^{2}+j^{2}+\alpha^{2}\right) \text {. }
$$

The transport components (hu, hv) can, of course, be recovered from the solution for $\psi$ by the diagnostic relations $(u h, v h)=\left(-\psi_{y}, \psi_{x}\right)$.

For the topographic wave test problems, the following nominal values for the dimensional parameters have been used: 


$$
\begin{array}{lll}
\text { (i) along-channel length } & \mathrm{L}_{x}=10^{8} \mathrm{~cm}=1000 \mathrm{~km} \\
\text { (ii) cross-channel length } & \mathrm{L}_{y}=5 \times 10^{7} \mathrm{~cm}=500 \mathrm{~km} \\
\text { (iii) depth at } y=0 & H_{0}=5 \times 10^{4} \mathrm{~cm}=500 \mathrm{~m} \\
\text { (iv) } & \text { Coriolis parameter } & \mathrm{f}_{0}=1.0 \times 10^{-4} \mathrm{sec}^{-1} \\
\text { (v) } & \text { constant density } & \rho \equiv 1 \mathrm{gm} \mathrm{cm}^{-3} .
\end{array}
$$$$
\text { (iii) depth at } y=0
$$

and

(v) constant density

The choice of a specific analytic solution $\psi_{a}(x, y, t)$ involves the specification of three physical parameters. These are: the along-channel wavenumber, $k$; the cross-channel mode number, $j$; and the topographic e-folding scale length, $\alpha$. Having chosen these parameters, the frequency of the wave follows from (34).

In ascertaining the accuracy with which the numerical model recovers the nonlinear wave solutions (33), the important non-dimensional computational parameters are:

(i) (Number of points in $x / x$ wavescale) $=L / k$

(ii) (Number of points in $y / y$ mode scale) $=M k / j$ or $M / \alpha L_{y}$

and (iii) ( $\Delta t /$ wave period) $=\Delta t \omega / 2 \pi$.

Since the analytic solution is depth-invariant, there is no obvious meaningful measure of the formal accuracy of the $\sigma$ expansion. However, it will be important to note any spurious generation of higher modes $(k>0)$ by the numerical model; since these modes identically vanish for the analytic solution, we hope that they remain small in the model solution.

Table I shows the results of these analytic tests. As well as the relevant parameter values, the table lists the normalized RMS differences between the model-generated and analytic wave solutions for $\psi, u$ and $v$ 
after an elapsed time of one wave period. Here, the normalized RMS error in $\psi$ is defined as

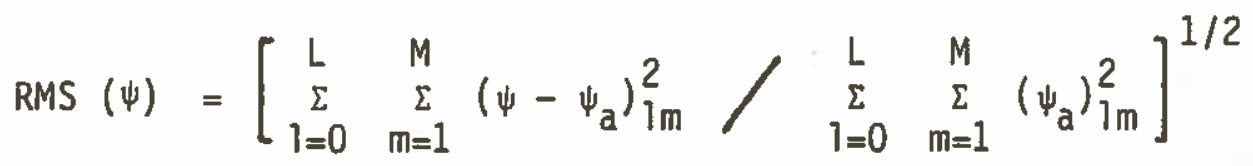

with similar definitions for $\operatorname{RMS}(u)$ and $\operatorname{RMS}(v)$.

For these wave problems and the range of parameters studied, RMS errors are generally about one percent after one wave period. Model errors depend most sensitively on the non-dimensional time-step. (Compare, for instance, cases 4 and 5 . Note the nearly quadratic dependence of RMS error on $\Delta t$.$) The relative insensitivity of RMS error to the effective x$ resolution is a reflection of the high accuracy of the Fourier expansion technique. In particular, it is well known that Fourier simulations of wave propagation are free from any phase error attributable to space differencing for any resolved wave (Orszag and Israeli, 1974). While this is certainly not true of the second-order finite-difference representation in the $y$-direction, the analytic solutions examined here have phase propagation in $x$ only. Since the cross-channel modal structure is invariant in time, RMS errors are only weakly dependent on the cross-channel resolution parameters. An examination of cases 1, 4 and 6 indicates, however, that $\triangle y$-related RMS errors comparable to those arising from time-differencing begin to appear for these wave solutions when $(M / j)$ approaches $0(10)$. (Compare also cases 7 and 8.) Lastly, we note that the simulated wave solutions remain highly depth-invariant throughout the experiments. Higher mode amplitudes are typically smaller by four orders of magnitude (nearly machine accuracy) than the barotropic mode. 
These topographic wave tests have been run at the Woods Hole Oceanographic Institution on a VAX11-780 system. Calculations were carried out primarily in-core, with a minimum of "paging". The required cpu time per model time step is shown in Table II as a function of spatial resolution. These figures indicate that execution time increases roughly linearly with the number of horizontal grid points, and perhaps slightly more rapidly with the number of vertical expansion functions. To put these cpu times in an appropriate perspective, a two-month simulation using a $(32 \times 49 \times 5)$ grid, and a time step of 0.1 day would require roughly 3.5 cpu hours on the VAX, and much less on a more powerful machine. Even on a machine like the VAX, however, this cpu requirement is modest.

Prototype test problems characterized by non-trivial vertical structure have also been run, with roughly comparable error levels. Taken together, these results indicate that the four-dimensional model described here can be used to provide accurate and efficient numerical exploration of coastal/deep ocean coupling and physics.

\section{Acknowledgements}

The development of this model has been supported by the National Aeronautics and Space Administration (Grant NAG 5-8). 


\section{TABLE I}

Normalized RMS Model Errors in Streamfunction $(\psi)$ and Velocity $(u, v)$ After One Period for Nonlinear Topographic Wave Solutions

\begin{tabular}{lllllllllllll}
\hline Case & $L_{y} \alpha\left(M / \alpha L_{y}\right)$ & $j(M / j)$ & $k(L / k)$ & $L$ & $M$ & $N$ & $\frac{\Delta t W}{2 \pi}$ & $\begin{array}{c}\text { RMS } \psi \\
(\%)\end{array}$ & $\begin{array}{c}\text { RMS } \\
(\%)\end{array}$ & $\begin{array}{c}\text { RMS } \\
(\% / .)\end{array}$ \\
\hline 1 & $1.0(49)$ & $1(49)$ & $4(4)$ & 16 & 49 & 5 & 0.01 & 1.07 & 1.06 & 1.07 \\
2 & $1.0(25)$ & $1(25)$ & $4(4)$ & 16 & 25 & 5 & 0.01 & 1.14 & 1.14 & 1.14 \\
3 & $1.0(49)$ & $1(49)$ & $4(8)$ & 32 & 49 & 5 & 0.01 & 1.19 & 1.33 & 1.61 \\
4 & $1.0(49)$ & $2(25)$ & $4(4)$ & 16 & 49 & 5 & 0.01 & 1.26 & 1.26 & 1.26 \\
5 & $1.0(49)$ & $2(25)$ & $4(4)$ & 16 & 49 & 5 & 0.02 & 4.46 & 4.41 & 4.46 \\
6 & $1.0(49)$ & $4(12)$ & $4(4)$ & 16 & 49 & 5 & 0.01 & 2.94 & 3.08 & 2.94 \\
7 & $2.0(25)$ & $2(25)$ & $4(4)$ & 16 & 49 & 5 & 0.01 & 1.34 & 1.33 & 1.33 \\
8 & $2.0(13)$ & $2(13)$ & $4(4$ & 16 & 25 & 5 & 0.01 & 2.19 & 2.53 & 2.17 \\
9 & $1.0(25)$ & $2(13)$ & $4(4)$ & 16 & 25 & 10 & 0.01 & 1.88 & 2.30 & 1.88
\end{tabular}


TABLE I I

Required cpu Time Per Mode1 Time-Step on a VAX11-780

\begin{tabular}{llrr}
\hline$L$ & $M$ & $N$ & \multicolumn{2}{c}{$\begin{array}{r}\text { Seconds cpu } \\
\text { Per Time Step }\end{array}$} \\
\hline 16 & 25 & 5 & 5.9 \\
16 & 49 & 5 & 11.9 \\
32 & 49 & 5 & 21.8 \\
16 & 25 & 10 & 13.9 \\
\hline
\end{tabular}


APPENDIX

DETAILS OF THE CHEBYSHEV-TAU SOLUTION TECHNI QUE

Recall from Section 4 that the vertical (o) dependence of the dependent variables $(u, v, p, w)$ has been represented as an expansion in the modified Chebyshev basis function $P_{k}(\sigma)$, where

$$
P_{k}(\sigma)= \begin{cases}T_{0}(\sigma) & k=0 \\ T_{0}(\sigma) & k>0, \text { odd } \\ T_{0}(\sigma)+\left[\frac{1}{k^{2}-1}\right] & k>0, \text { even }\end{cases}
$$

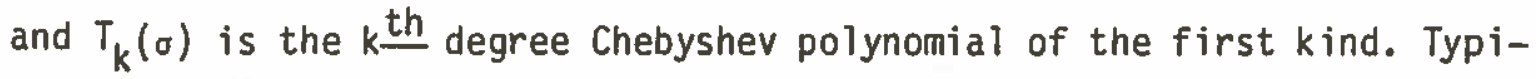
cally defined over the interval $-1 \leq \sigma \leq 1, T_{k}(\sigma)=\cos \left[k \cos ^{-1}(\sigma)\right]$ where $-\pi \leq \cos ^{-1}(0) \leq 0$. For the purposes of the following discussion, we introduce an arbitrary one-dimensional variable $n$, and set

$$
n_{n}=n\left(\sigma_{n}\right)=\sum_{k=0}^{N} n_{k} T_{k}\left(\sigma_{n}\right) \quad 0 \leq n \leq N .
$$

The "collocation" points or equivalent gridpoints, ${ }_{n}$, are conveniently chosen to correspond to the extrema of $T_{N}(\sigma)$; hence,

$$
\sigma=\cos [\pi(n-N) / N] . \quad 0 \leq n \leq N
$$

Given $\eta\left(\sigma_{n}\right)$ at the $(N+1)$ collocation points $\sigma_{n}(0 \leq n \leq N)$, the equiva lent expansion coefficients $\eta_{k}(0 \leq k \leq N)$ can be recovered in several ways. For instance, a Fast Cosine Transform (FCT) can be used - see, e.g., Haidvogel and Zang (1979). Alternately, a linear (matrix) transformation can be constructed to compute the $n_{k}$ from $n_{n}$. The required transformation matrix $F$ is given by 


$$
F=\left(\begin{array}{cccc}
P_{0}(-1) & P_{1}(-1) & \ldots & P_{N}(-1) \\
P_{0}\left(\sigma_{1}\right) & P_{1}\left(\sigma_{1}\right) & \ldots & P_{N}\left(\sigma_{1}\right) \\
\vdots & & & \\
P_{0}(1) & P_{1}(1) & \ldots & P_{N}(1)
\end{array}\right) \text {. }
$$

Considering $n_{n}$ and $n_{k}$ to be column vectors, it is easily seen that

$$
F_{n_{k}}=n_{n} \quad 0 \leq n, k \leq N
$$

and, hence, that $\quad F^{-1} n_{n}=n_{k}$.

The identical approach can be used to form matrix operators which perform a vertical derivative or integral. For instance,

$$
\frac{d n}{d \sigma}\left(\sigma_{n}\right)=\delta_{\sigma} n_{n}=D n_{n}
$$

where

$$
D=\left(\begin{array}{cccc}
P_{0}^{\prime}(-1) & P_{1}^{\prime}(-1) & \ldots & P_{N}^{\prime}(-1) \\
P_{0}^{\prime}\left(\sigma_{1}\right) & P_{1}^{\prime}\left(\sigma_{1}\right) & \ldots & P_{N}^{\prime}\left(\sigma_{1}\right) \\
\vdots & & & \\
P_{0}^{\prime}(1) & P_{1}^{\prime}(1) & \ldots & P_{N}^{\prime}(1)
\end{array}\right) F^{-1}
$$

and

$$
\begin{aligned}
P_{k}^{\prime}(\sigma) & =\frac{d}{d \sigma} P_{k}(\sigma)=\frac{d}{d \sigma} T_{k}(\sigma) \\
& =\frac{n \sin (n \theta)}{\sin \theta} \quad \theta=\cos ^{-1}(\sigma) .
\end{aligned}
$$

Likewise,

$$
\int_{\sigma_{n}}^{1} P(\sigma) d \sigma=I_{\sigma_{n}}^{1}(P)=S n_{k}
$$


where

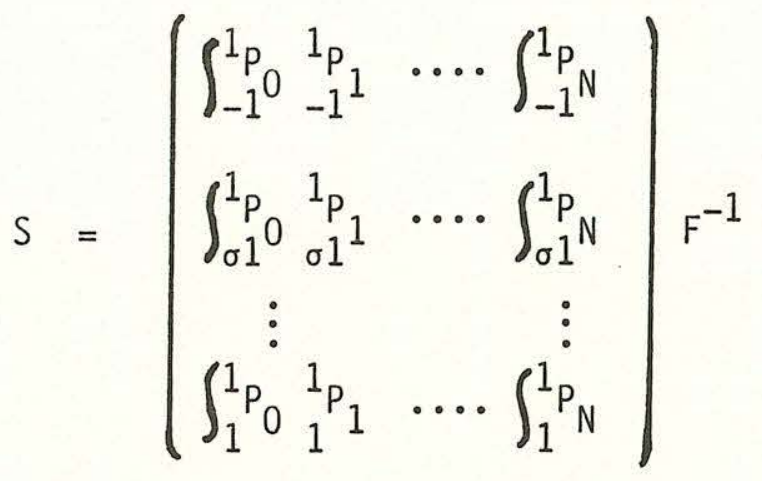

and

$$
\int_{\sigma_{n}}^{1} P_{k}(\sigma) d \sigma= \begin{cases}\frac{1}{2}\left(1-\sigma_{n}\right) & k=0 \\ \frac{1}{2}\left(1-\sigma_{n}^{2}\right) & k=1 \\ \left.\left(\frac{\cos (n+1) x}{2(n+1)}-\frac{\cos (n-1) x}{2(n-1)}\right)\right|_{\cos ^{-1}\left(\sigma_{n}\right)} ^{0} & \quad k>1, \text { odd } \\ \left.\left(\frac{\cos (n+1) x}{2(n+1)}-\frac{\cos (n-1) x}{2(n-1)}\right)\right|_{\cos ^{-1}\left(\sigma_{n}\right)} & \text { (A.12) } \\ +\left(1-\sigma_{n}\right) \frac{1}{\left(k^{2}-1\right)} & k>1 \text {, even }\end{cases}
$$

As described in Section 4, the tau approximation replaces the dynamic equations for $\mathrm{k}=\mathrm{N}-1, \mathrm{~N}$ - that is, those which dictate the dynamical evolution of the highest two vertical modes - with the two boundary conditions at top and bottom $(\sigma= \pm 1)$. The implementation of the tau method proceeds as follows. Suppose that our time-stepping scheme has provided values of $n_{k}^{q+1}(0 \leq k \leq N-2)$, but that our boundary conditions require that

$$
n(-1)=0
$$


and

$$
\frac{d \eta}{d \sigma}(1)=\gamma
$$

Then,

$$
\left[\begin{array}{ll}
P_{N-1}(-1) & P_{N}(-1) \\
P_{N-1}^{\prime}(1) & P_{N}^{1}(1)
\end{array}\right]\left[\begin{array}{c}
n_{N-1}^{q+1} \\
\eta_{N}^{q+1}
\end{array}\right]=\left[\begin{array}{c}
-\hat{n}(-1) \\
\gamma-\frac{d \hat{n}}{d \sigma}(1)
\end{array}\right]
$$

where $\quad \hat{n}_{k}=\left\{\begin{array}{ll}n_{k}^{q+1} & k=0, \ldots, N-2 \\ 0 & k=N-1, N\end{array}\right.$.

The algebraic equation (A.13) is readily solved. Note that the same sequence of steps leading to (A.13, A.14) yield similar simple expressions for $n_{k}(k=N-1, N)$ for various alternate top and bottom boundary conditions. 


\section{References}

Beardsley, R. C. and C. D. Winant, 1979. On the mean circulation in the Mid-Atlantic Bight. $\mathrm{J}_{\text {. of Phys. Oceanog., }}$, , 612-619.

Csanady, G. T., 1978. The arrested topographic wave. J. Phys. Oceanog., 8, $47-62$.

Freeman, N. G., A. M. Hale, and M. B. Danard, 1972. A modified sigma equations' approach to the numerical modeling of Great Lakes hydrodynamics. J. Geophys. Res., 77, 1050-1060.

Gottlieb, D. and S. A. Orszag, 1977. Numerical Analysis of Spectral Methods: Theory and Applications. CBMS-NSF Monograph Series, Society for Industrial and Applied Mathematics.

Haidvogel, D. B., 1979. A discussion of certain modeling factors which influence the results of eddy-resolving ocean circulation studies. Dyn. Atmos. Oceans, $\underline{3}, 181-190$.

Haidvogel, D. B. and T. Zang, 1979. The accurate solution of Poisson's equation by expansion in Chebyshev polynomials. J. Comp. Phys., 30 , 167-180.

Krol1, J. and P. P. Niiler, 1976. The transmission and decay of barotropic topographic Rossby waves incident on a continental shelf. J. Phys. 0ceanog., $\underline{6}, 432-450$.

Orszag, S. A. and M. Israeli, 1974. Numerical simulation of viscous incompressible flows. Ann. Rev. Fluid Mech., 6, 281-318.

Ou, H. -W., 1979. On the propagation of free topographic Rossby waves near continental margins. Ph.D. Dissertation, Massachusetts Institute of Technology and the Woods Hole Oceanographic Institution.

Owen, A., 1980. A three-dimensional model of the Bristol Channel. J. Phys. Oceanog., 10, 1290-1302.

Phillips, N. A., 1957. A coordinate system having some special advantages for numerical forecasting. J. Meteor., 14, 184-185.

Ring Workshop, 1977. Oceanographic Study of Warm Core Gulf Stream Rings and the Northwest Atlantic Slope Water Region. Report and Proceedings of the Interdisciplinary Workshop on Gulf Stream Anticyclonic Eddies; Woods Hole, May, 1977.

Smith, P. C., in press. Eddies and coastal interaction. Chapter 20 in: Eddies in Marine Science; A. R. Robinson, editor.

Thompson, R.0.R.Y., 1977. Observations of Rossby waves near Site D. Progress in Oceanography, $\underline{7}, 1-28$. 


\begin{tabular}{|c|c|c|}
\hline $\begin{array}{l}\text { REPORT DOCUMENTATION } \\
\text { PAGE }\end{array}$ & 1. REPOHT MO. WHOI-81-90 & 3. Aoctplent'z Accession No. \\
\hline \multirow{2}{*}{\multicolumn{2}{|c|}{$\begin{array}{l}\text { 4. Thete and subtitie } \\
\text { A FOUR-DIMENSIONAL PRIMITIVE EQUATION MODEL. FOR COUPLED } \\
\text { COASTAL- DEEP OCEAN STUDIES }\end{array}$}} & $\begin{array}{l}\text { 5. Roport Doto } \\
\text { October } 1987\end{array}$ \\
\hline & & 6. \\
\hline \multicolumn{2}{|l|}{$\begin{array}{l}\text { 7. Author(a) } \\
\text { Dale B. Haidvogel }\end{array}$} & a. Parforming Orgenlzetion Roph. No. \\
\hline \multirow{3}{*}{\multicolumn{2}{|c|}{$\begin{array}{l}\text { Portomina Orentratlon Name and Addrous } \\
\text { Woods Hole Oceanographic Institution } \\
\text { Woods Hole, Massachusetts } 02543\end{array}$}} & 10. Prolect/Tosk/Wark Unit No. \\
\hline & & $\begin{array}{l}\text { 11. Contract(C) or Grant(G) No. } \\
\text { (C) }\end{array}$ \\
\hline & & (G) NAG 5-8 \\
\hline \multirow{3}{*}{\multicolumn{2}{|c|}{$\begin{array}{l}\text { 12. Sponserine Organlzatlon Mame and Addrons } \\
\text { The National Aeronautics and Space Administration } \\
\text { Washington, D.C. }\end{array}$}} & 12. Type of Ropert \& Parlod Covered \\
\hline & & Technical \\
\hline & & 14. \\
\hline
\end{tabular}

15. Supplamentery Notes

This report should be cited as: Woods Hole Oceanog. Inst. Tech. Rept. WHOI-81-90.

16. Abatrect (Limit: 200 words)

A prototype four-dimensional $(x, y, z, t)$ continental shelf/deep ocean model is described. In its present form, the model incorporates the effects of finite-amplitude topography, advective nonlinearities, and variable stratification and rotation. The model can be forced either directly by imposed atmospheric windstress and surface pressure distributions, and energetic mean currents imposed by the exterior oceanic circulation; or indirectly by initial distributions of shoreward propagating mesoscale waves and eddies.

To avoid concerns over the appropriate specification of "open" boundary conditions on the cross-shelf and seaward model boundaries, a periodic channel geometry (oriented along-coast) is used. The model employs a traditional finite-difference expansion in the cross-shelf direction, and a Fourier (periodic) representation in the long-shelf coordinate. A modified sigma coordinate system, and a Chebyshev-tau approximation scheme, are used to incorporate the vertical dependence.

The model has been validated against a variety of propagating topographic wave problems. Representative run times and error estimates are given.

17. Document Anviysi a. Descriptor

1. Coastal ocean model

2. Coastal/deep ocean coupling

3. Continental margin transport processes

b. Identlfiers/Open-Ended Terms

c. Cosati fleld/Group

10. Avellebility stetement

19. Security Clase (This Report)

Unclassifíed.

20. Securlty cless (Thls Pace)
21. No. of Pages

28

22. Price 


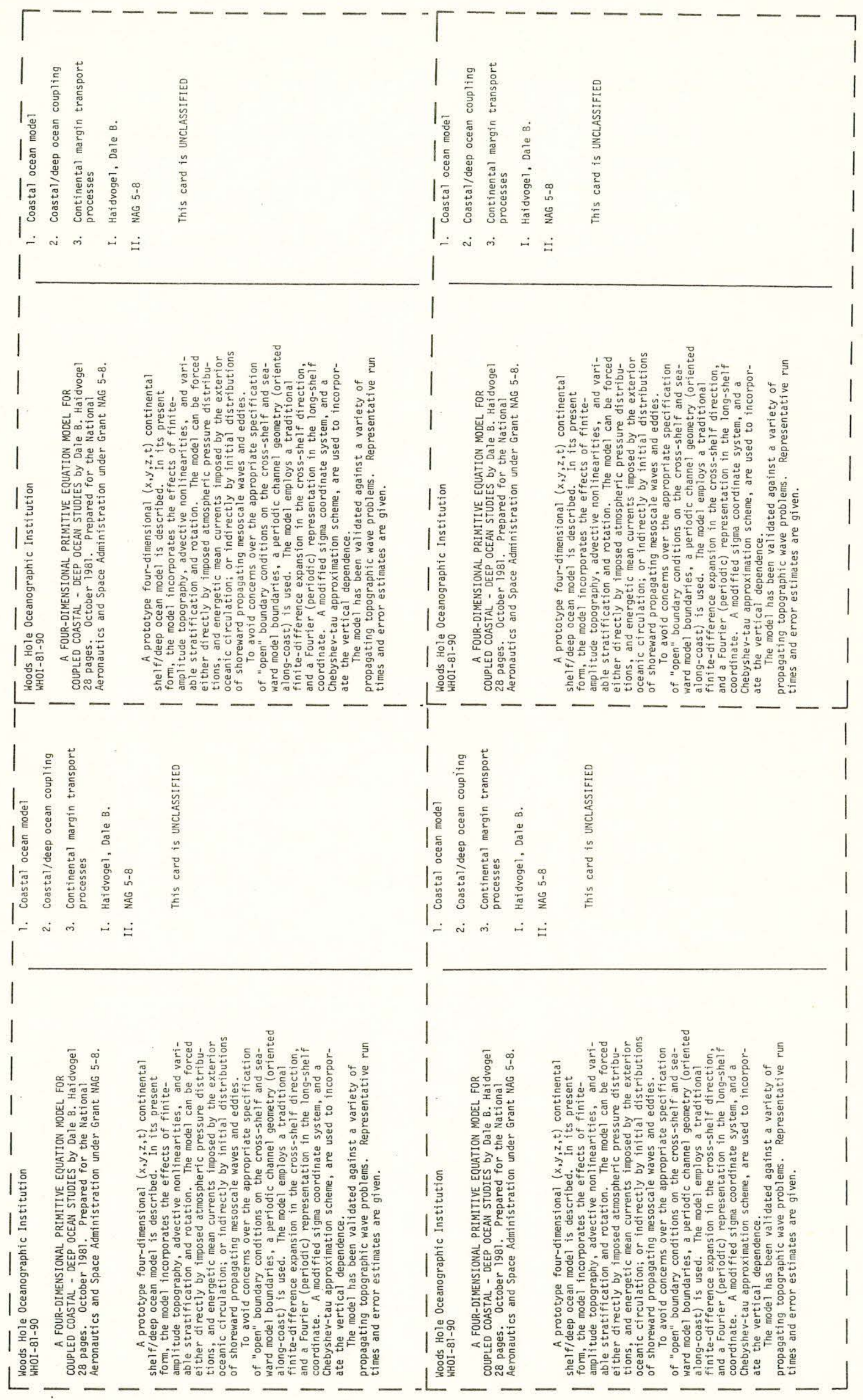

